Psychother Psychosom 2019;88:58-60

DOI: $10.1159 / 000495044$

\title{
Weight Gain after Fecal Microbiota Transplantation in a Patient with Recurrent Underweight following Clinical Recovery from Anorexia Nervosa
}

\author{
Nicolien C. de Clercq ${ }^{\mathrm{a}}$ Myrthe N. Frissen ${ }^{\mathrm{a}}$ Mark Davids $^{\mathrm{a}}$ \\ Albert K. Groen ${ }^{\mathrm{a}, \mathrm{b}}$ Max Nieuwdorp ${ }^{\mathrm{a}, \mathrm{c}}$ \\ a Department of Internal and Vascular Medicine, Amsterdam \\ University Medical Center, Amsterdam, The Netherlands; \\ ${ }^{b}$ Department of Pediatrics, University Medical Center \\ Groningen, University of Groningen, Groningen, The \\ Netherlands; ' Wallenberg Laboratory, University of Gothenburg, \\ Gothenburg, Sweden
}

Anorexia nervosa (AN) is a life-threatening eating disorder characterized by a distorted body image and excessive dieting, leading to severe underweight and concomitant mental and somatic comorbidities. Weight restoration is an important treatment target since it prevents deterioration and improves patients' overall prognosis, but effective strategies to achieve this goal are lacking. Especially in the restricting type, underweight can be resistant despite hypercaloric diets [1].

The gut microbiome has recently been identified as one of the regulators of human bodyweight. Fecal microbiota transplantation (FMT) from obese to nonobese subjects has led to weight gain despite unchanged dietary intake in animals as well as humans [2]. It has been repeatedly shown that short- and long-term dietary patterns including undernutrition and dieting in patients with AN can change the microbiota composition [3]. The notion that the gut microbiome can also affect nervous system functions through the gut-brain axis makes its involvement in eating behavior and psychiatric disorders plausible [4].

Accordingly, we present a case in which FMT led to weight gain in a 26-year-old female after unsuccessful attempts to maintain a healthy bodyweight following clinical recovery from AN (restricting type), confirmed by a clinical psychiatrist at an eating disorder center (PsyQ in Groningen). The disease had presented 2 years earlier, and the body mass index (BMI) increased from 14 to 19 with cognitive-behavioral therapy, transient psychotropic medication, and short-term force feeding. Despite the maintenance of a hypercaloric diet of 2,500 kcal/day and a desire to maintain a healthy weight, her BMI dropped and stabilized at 15 after discharge. Except for a stable regime of prednisolone and methotrexate for preexisting autoimmune dermatomyositis, she had not used any medication or supplements since discharge (a year before presentation to our clinic). Her medical history was otherwise un-

\section{KARGER}

E-Mail karger@karger.com www.karger.com/pps
(C) 2019 The Author(s)

Published by S. Karger AG, Basel

Karger

Open access

This article is licensed under the Creative Commons AttributionNonCommercial-NoDerivatives 4.0 International License (CC BY NC-ND) (http://www.karger.com/Services/OpenAccessLicense) Usage and distribution for commercial purposes as well as any distribution of modified material requires written permission. remarkable, with no cholecystectomy, gastrointestinal surgery, or other events known to influence gut microbial composition. Importantly, she did not adhere to a specific diet, smoke, or drink alcohol (confirmed through dietary lists).

FMT was successfully performed according to protocol (online suppl. material; for all online suppl. material, see www.karger.com/ doi/10.1159/000495044) with feces from an unrelated healthy female donor with a BMI of 25. Dietary intake was reported through online application 7 days prior to each visit. Changes in metabolic parameters (resting metabolism and bodyweight) and body composition (body fat and lean mass) were assessed at three time points (baseline, 6, 12, and 36 weeks; Fig. 1a).

Over this period, the patient gained $6.3 \mathrm{~kg}$ in bodyweight (from 45.8 to $52.1 \mathrm{~kg}$ ), mostly due to a $55 \%$ increase in body fat and despite a reported stable caloric intake. Interestingly, resting energy expenditure was decreased on all post-measurements compared to baseline. Gut microbial composition showed an increase in weighted phylogenetic diversity at 6 and 12 weeks with an especially marked increase in the number of Verrucomicrobia (Fig. 1b). As expected from previous FMT studies, the gut microbiota composition slowly changed back towards the patients' initial personal core microbial composition [5]. No side effects from FMT were reported or observed during the entire study period.

In this patient, FMT resulted in a $13.8 \%$ weight gain over a $36-$ week period, which could have various explanations. One often proposed hypothesis is the varying degree to which certain microbes can extract calories from food, for example through the production of short-chain fatty acids (SCFAs) from indigestible carbohydrates (see below), resulting in an increased extraction of energy despite stable dietary intake [6]. Furthermore, studies have assigned a role for gut microbes in host metabolic rates, the latter of which was found altered in AN patients [3]. In this case, the resting energy metabolism decreased after FMT, possibly contributing to the gain in bodyweight.

The phyla Firmicutes, Bacteroidetes, and, to a lesser extent, Verrucomicrobia and Euryarchaeota were the dominant phyla in both patient and donor. However, the patient and donor showed profound differences with respect to the relative abundance of these bacterial phyla. In line with previous studies, the relative abundance of Bacteroidetes in the patient was significantly lower and Firmicutes levels higher at baseline compared to the healthy donor [7].

Furthermore, there were significant differences in gut microbial composition after FMT, with the introduction of the Akkermansia muciniphila species (Verrucomicrobia in Fig. 1b). As a mucin degrader, higher levels of A. muciniphila have repeatedly been associated with improved metabolic health, and regression towards an optimum bodyweight for both obese as well as underweight individuals [6]. The effect is thought to be mediated through the production of SCFAs as well as cross-feeding other beneficial microbes with mucin-degradation products like oligosaccharides.
Nicolien C. de Clercq

Department of Internal and Vascular Medicine Amsterdam University Medical Center NL-1105 AZ Amsterdam (The Netherlands)

E-Mail n.c.declercq@amc.uva.nl 

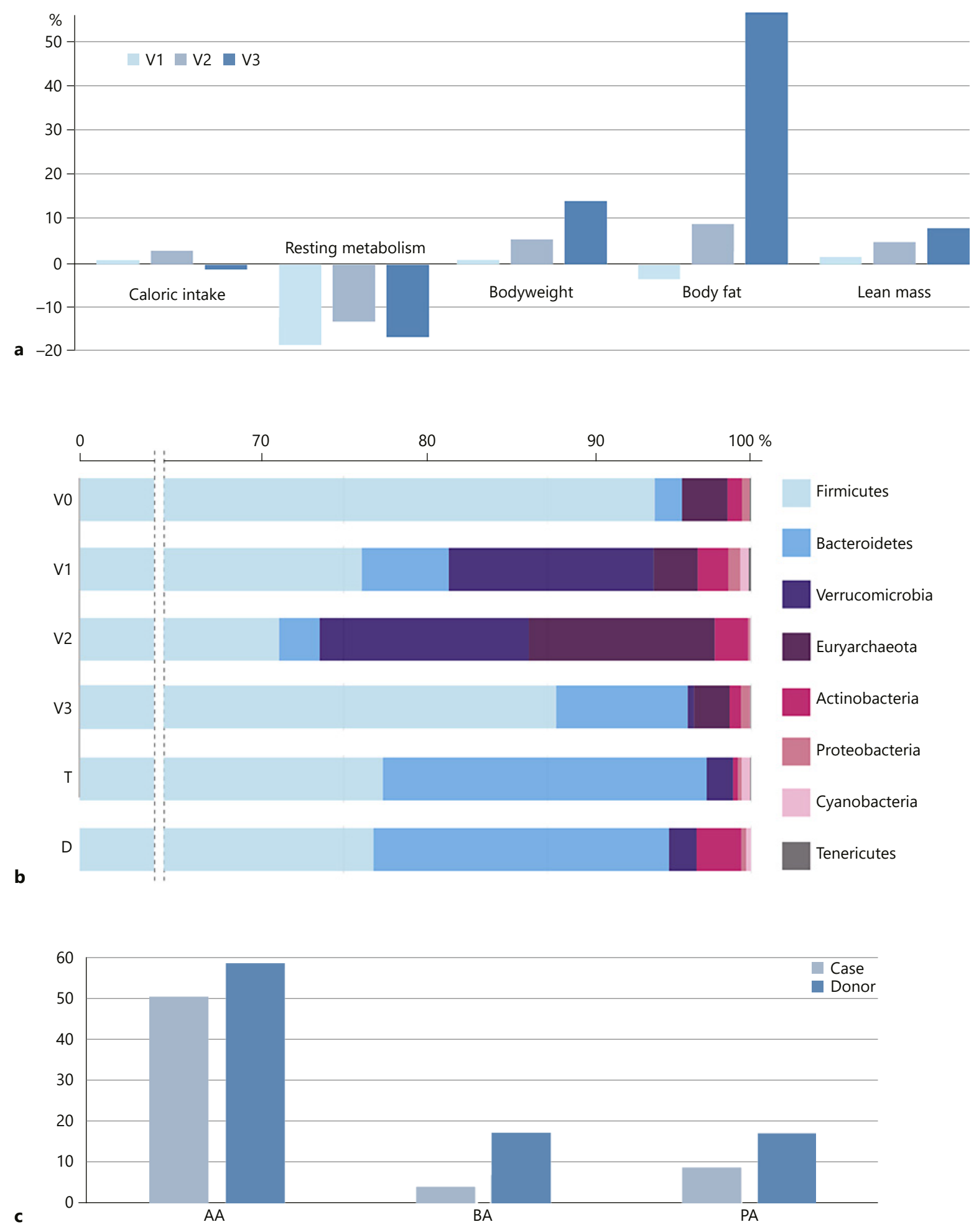

Fig. 1. Effect of FMT. a Percentage change in caloric intake, resting energy metabolism, bodyweight (kg), body fat (kg), and lean mass (kg) at 6 weeks (V1), 12 weeks (V2), and 36 weeks (V3) compared to baseline (V0). b Gut microbiota composition. T, composition of microbial transplant; D, composition of donor gut microbiota. c Percentage change in SCFAs compared to baseline. AA, acetate; BA, butyrate; PA, propionate.

Weight Gain after FMT following Recovery from AN 
Our results show increased SCFA levels in the donor sample compared to the patient at baseline (Fig. 1c). FMT induced a significant increase in both fecal acetate and butyrate levels in the recipient, which might explain the change in metabolic rate. However, in contrast to our findings, an observational study performed in patients with AN showed no recovery of SCFA profiles upon weight gain [7].

While this case is illustrative of the therapeutic potential of FMT in AN, it is too early to claim causality since the interpretation of the results is hindered by limitations inherent to the study design. Firstly, the information on dietary intake depended on selfreports which are known to be subjected to bias, yet in this case it was considered the most feasible method due to practical and ethical reasons. Also, information on changes in physical activity or physical energy expenditure was not available.

Furthermore, even though medical interventions did not change during the study period, we cannot predict whether the regimen of prednisolone and methotrexate altered the outcomes from FMT in this individual case. As a final remark, although these results are promising, we should take into account that FMT is also associated with adverse events, like diarrhea, constipation, and infections [8].

In conclusion, we showed for the first time that FMT induced weight gain in a patient with recurrent $\mathrm{AN}$, suggesting that gut dysbiosis may be one of the causal factors in the etiology of persistent underweight in AN. To prove this possible connection, our group has initiated a randomized controlled trial evaluating the effects of FMT in underweight individuals suffering from AN (see online suppl. material). FMT could potentially overcome the resistant microbial signatures and consequent metabolic and clinical features upon sole nutritional rehabilitation for AN patients, and thus facilitate nutritional rehabilitation [7]. Especially considering the high recurrence and mortality rates, establishing additional therapeutic options are of essence for improved patient care in the field of AN. However, caution is required and it is important to recognize that FMT will never replace the standard multimodality treatment in patients with AN.
Disclosure Statement

M. Nieuwdorp is a member of the Scientific Advisory Board of Caelus Pharmaceuticals, the Netherlands.

Funding Sources

M. Nieuwdorp is supported by a ZONMW-VIDI grant 2013 (016.146.327) and CVON Young Talent grant 2012.

\section{References}

1 Marzola E, Nasser JA, Hashim SA, Shih PA, Kaye WH. Nutritional rehabilitation in anorexia nervosa: review of the literature and implications for treatment. BMC Psychiatry. 2013;13(1):290.

2 Ridaura VK, Faith JJ, Rey FE, Cheng J, Duncan AE, Kau AL, et al. Gut microbiota from twins discordant for obesity modulate metabolism in mice. Science. 2013 Sep;341(6150):1241214

3 Kleiman SC, Watson HJ, Bulik-Sullivan EC, Huh EY: The intestinal microbiota in acute anorexia nervosa and during renourishment: relationship to depression, anxiety, and eating disorder psychopathology. Psychosom Med. 2015;77(9):969-81.

4 Herpertz-Dahlmann B, Seitz J, Baines J. Food matters: how the microbiome and gut-brain interaction might impact the development and course of anorexia nervosa. Eur Child Adolesc Psychiatry. 2017 Sep;26(9):103141.

5 Turnbaugh PJ, Hamady M, Yatsunenko T, Cantarel BL, Duncan A, Ley $\mathrm{RE}$, et al. A core gut microbiome in obese and lean twins. Nature. 2009 Jan;457(7228):480-4.

6 Canfora EE, Jocken JW, Blaak EE. Short-chain fatty acids in control of body weight and insulin sensitivity. Nat Rev Endocrinol. 2015 Oct; 11(10):577-91.

7 Mack I, Cuntz U, Grämer C, Niedermaier S, Pohl C, Schwiertz A, et al. Weight gain in anorexia nervosa does not ameliorate the faecal microbiota, branched chain fatty acid profiles, and gastrointestinal complaints. Sci Rep. 2016 May;6(1):26752.

8 Agrawal M, Aroniadis OC, Brandt LJ, Kelly C, Freeman S, Surawicz C, et al. The long-term efficacy and safety of fecal microbiota transplant for recurrent, severe, and complicated clostridium difficile infection in 146 elderly individuals. J Clin Gastroenterol. 2016 May-Jun;50(5):403-7. 